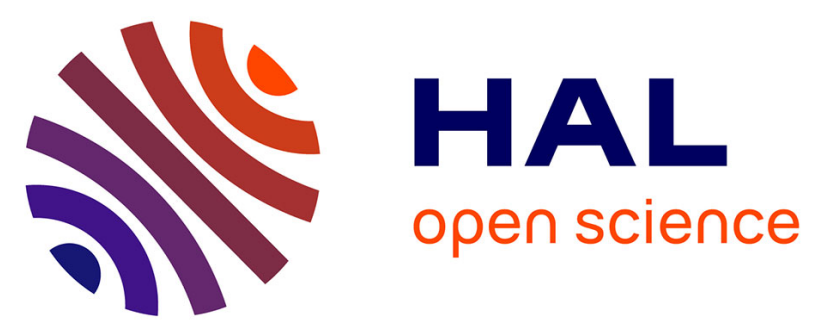

\title{
QEPAS Sensor Based on the Tracking of the Photoacoustic Induced Frequency Shift of a Tuning Fork Maintained in Self-Sustained Oscillation by Electrical Excitation
}

Maxime Duquesnoy, Raphaël Lévy, Jean-Michel Melkonian, Guillaume Aoust, Myriam Raybaut, Antoine Godard

\section{To cite this version:}

Maxime Duquesnoy, Raphaël Lévy, Jean-Michel Melkonian, Guillaume Aoust, Myriam Raybaut, et al.. QEPAS Sensor Based on the Tracking of the Photoacoustic Induced Frequency Shift of a Tuning Fork Maintained in Self-Sustained Oscillation by Electrical Excitation. CLEO: Science and Innovations 2021, May 2021, San Jose, United States. pp.SM4N.7, 10.1364/CLEO_SI.2021.SM4N.7 . hal-03480361

\section{HAL Id: hal-03480361 https://hal.science/hal-03480361}

Submitted on 14 Dec 2021

HAL is a multi-disciplinary open access archive for the deposit and dissemination of scientific research documents, whether they are published or not. The documents may come from teaching and research institutions in France or abroad, or from public or private research centers.
L'archive ouverte pluridisciplinaire HAL, est destinée au dépôt et à la diffusion de documents scientifiques de niveau recherche, publiés ou non, émanant des établissements d'enseignement et de recherche français ou étrangers, des laboratoires publics ou privés. 


\title{
QEPAS Sensor Based on the Tracking of the Photoacoustic Induced Frequency Shift of a Tuning Fork Maintained in Self-Sustained Oscillation by Electrical Excitation
}

\author{
M. Duquesnoy ${ }^{1,2}$, R. Lévy ${ }^{2}$, J-M. Melkonian ${ }^{2}$, G. Aoust ${ }^{1}$, M. Raybaut ${ }^{2}$, A. Godard ${ }^{2}$ \\ ${ }^{1}$ Mirsense, Nanno-INNOV, building 863, 8 Avenue de la Vauve, 91120 Palaiseau, France \\ ${ }^{2}$ DPHY, ONERA, Université Paris Saclay, F-91123 Palaiseau, France \\ maxime.duquesnoy@mirsense.com
}

\begin{abstract}
Oscillator frequency shift induced by the photoacoustic force on a closed-loop electrically excited quartz tuning fork is exploited to carry out QEPAS measurement. We report recent improvements of this new method for background-free gas sensing. (C) 2020 The Author(s)
\end{abstract}

Recently, quartz tuning forks (QTF) have been used in photoacoustics in order to measure the acoustic waves emitted by the gas excitation and relaxation [1]. These resonators represent a further step towards sensitive and cheap gas sensors. In addition, due to their quadripolar nature and high quality factor $Q$, QTFs present a better immunity to external backgrounds (acoustic, vibratory) than traditional photoacoustic detectors such as microphones. Nonetheless, they also suffer from main disadvantages such as the drift of their frequency and quality factor over time, particularly due to temperature changes, making their use out of the laboratory difficult.

Several approaches were proposed to circumvent these issues, including beat frequency QEPAS [2] or by repeatedly tracking the frequency and quality factor of the QTF and measuring the gas concentration in a classic QEPAS detection scheme, however provoking periodic sensor dead time [3]. Yet other methods were proposed as the alternate electrical excitation of the QTF and measurement of its decay time [4]. Here, we present our last results with a new closed-loop detection method [5], which enables to get rid of background drifts without measurement dead time.

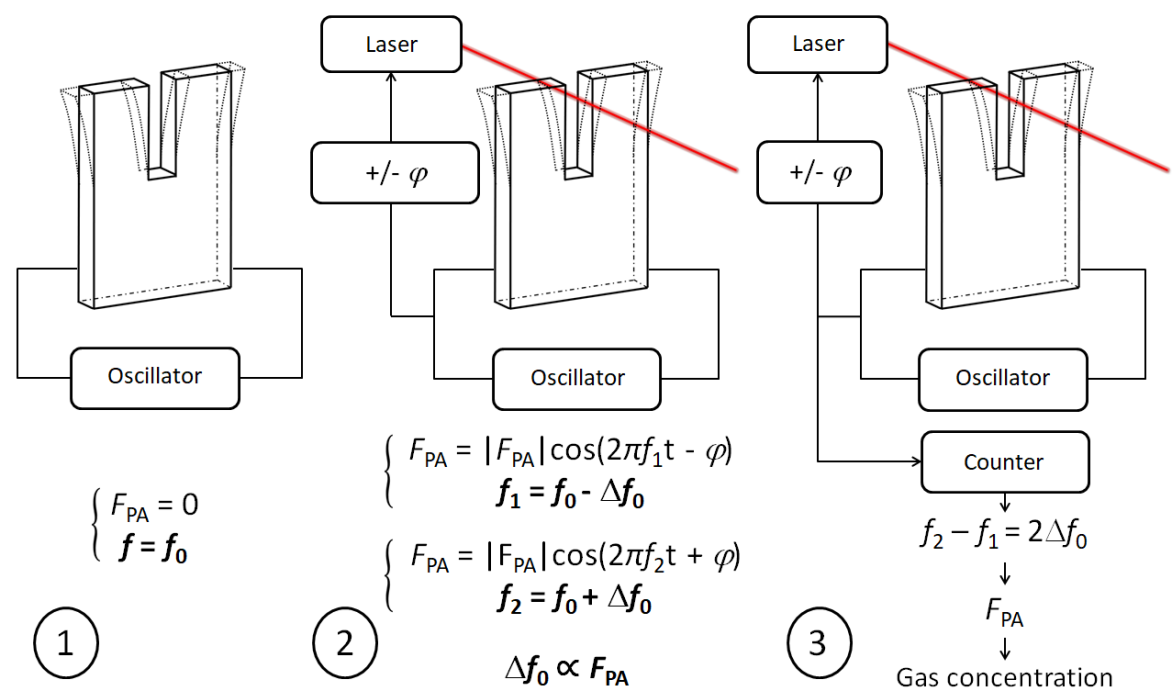

Fig. 1. Schematic of the measurement principle. The photoacoustic force induces a frequency shift that we measure to determine gas concentration.

While most photoacoustic devices use an open-loop detection scheme of the QTF signal, we introduce a closedloop detection scheme. The QTF is driven at its fundamental frequency $f_{0}$ by an external electrical oscillator inducing a force $F_{\mathrm{X}}$. When a photoacoustic force $F_{\mathrm{PA}}$ is applied and modulated with a phase $\varphi$, a frequency shift $\Delta f_{0}$ occurs. This frequency shift is directly proportional to the photoacoustic force which is in turn proportional to the gas concentration to determine. This principle is illustrated in Figure 1 and an analytical expression of the 
frequency shift is given in Equation 1.

$$
\Delta f_{0} \approx \frac{f_{0}}{2 Q} \frac{\left|F_{\mathrm{PA}}\right|}{\left|F_{\mathrm{X}}\right|} \sin (\varphi)
$$

Moreover, a differential measurement is set by modifying the phase shift $\varphi$ used for laser modulation to repeatedly reverse the sign of the frequency shift induced by the photoacoustic force while the frequency drift due to background is not affected. A differential frequency shift $\Delta f_{0, \text { diff }} \approx \frac{1}{Q} f_{0}\left|F_{\mathrm{PA}}\right| /\left|F_{X}\right|$ can thus be obtained, allowing compensation of the frequency drifts over time and reducing the external signal backgrounds seen by the QTF.

In order to validate this principle and its analytical modelling we performed extensive measurements. Photoacoustic measurements were performed by targeting $\mathrm{C}_{2} \mathrm{H}_{2}$ in $\mathrm{N}_{2}$ at $6,490.02 \mathrm{~cm}^{-1}$ with a telecom laser diode. We chose one of our custom QTF for these measurements, presenting a frequency of $21 \mathrm{kHz}$ and a quality factor of 8,000 [6]. We particularly studied the influence of system parameters (excitation voltage, motional resistance, bandwidth) on the generated signal and noise, for which a very good agreement was reached. Ultimately, we verified that the signal was proportional to the gas concentration. A good linear fit was obtained as shown in Figure 2 (left). We then verified that the limiting noise was the oscillator Leeson's noise. This is illustrated in Figure 2 (right) where we compared the Allan deviation of the classical method and of ours. The different slopes represent the phase Flicker and white noise and white frequency noise, confirming that the sensor noise is at its fundamental limit.

Finally, thanks to the differential, our method enables a sensitivity enhancement of a factor two compared to the open-loop detection, yielding a sensitivity of $3.3510^{-8} \mathrm{~W} \cdot \mathrm{cm}^{-1} \cdot \mathrm{Hz}^{-1 / 2}$ which is similar to state-of-the art sensitivity for bare QTFs.
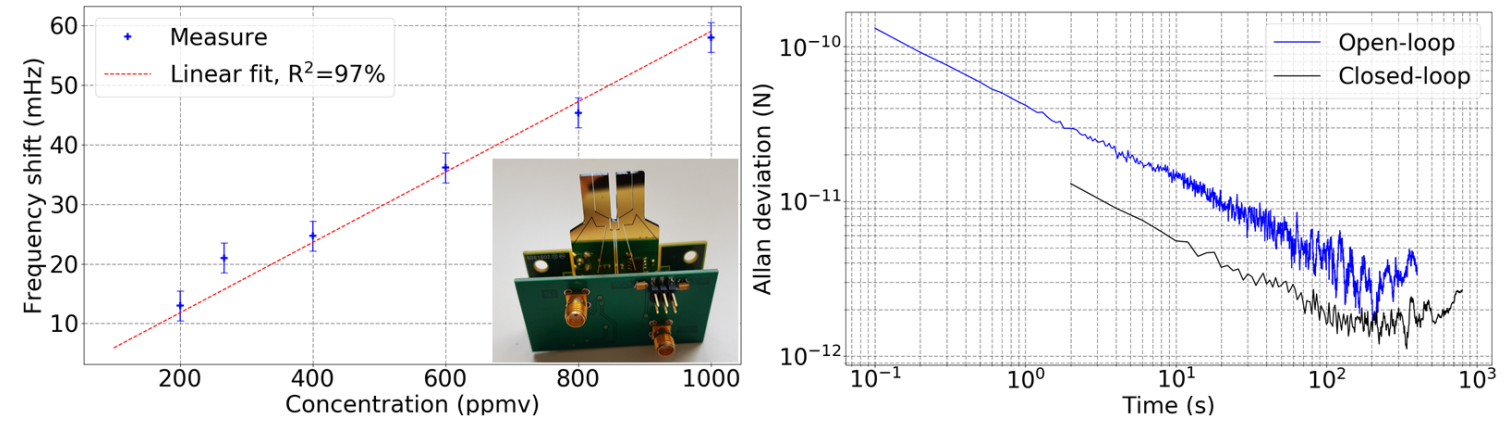

Fig. 2. Linearity with gas concentration and photography of the QTF and its electronics (left). Allan deviation of the signal equivalent force (right).

In the future, we will evaluate the long term stability of our system. We also strive to develop and integrate this new method in an industrial photoacoustic sensor.

\section{References}

1. Anatoliy A Kosterev, Yu A Bakhirkin, Robert F Curl, and Frank K Tittel. Quartz-enhanced photoacoustic spectroscopy. Optics letters, 27(21):1902-1904, 2002.

2. Hongpeng Wu, Lei Dong, Huadan Zheng, Yajun Yu, Weiguang Ma, Lei Zhang, Wangbao Yin, Liantuan Xiao, Suotang Jia, and Frank K Tittel. Beat frequency quartz-enhanced photoacoustic spectroscopy for fast and calibration-free continuous trace-gas monitoring. Nature communications, 8(1):1-8, 2017.

3. Roman Rousseau, Nicolas Maurin, Wioletta Trzpil, Michael Bahriz, and Aurore Vicet. Quartz tuning fork resonance tracking and application in quartz enhanced photoacoustics spectroscopy. Sensors, 19(24):5565, 2019.

4. M Mordmueller, W Schade, and U Willer. QEPAS with electrical co-excitation for photoacoustic measurements in fluctuating background gases. Applied Physics B, 123(8):224, 2017.

5. Raphael Levy, Maxime Duquesnoy, Jean-Michel Melkonian, Myriam Raybaut, and Guillaume Aoust. New signal processing for fast and precise QEPAS measurements. IEEE Transactions on Ultrasonics, Ferroelectrics, and Frequency Control, 67(6):1230-1235, 2019.

6. Maxime Duquesnoy, Guillaume Aoust, Jean-Michel Melkonian, Raphaël Lévy, Myriam Raybaut, and Antoine Godard. Quartz enhanced photoacoustic spectroscopy based on a custom quartz tuning fork. Sensors, 19(6):1362, 2019. 